\title{
((Contractible Edge of Eulerian Graph- Regular )) \\ https://doi.org/10.32792/utq/utj/vol11/4/3
}

\author{
Azhar Aziz Sangoor \\ Department of Mathematics \\ University of Thi-Qar \\ College science of Computer and mathematics \\ Azhar Aziz 1984@gmail.com
}

\begin{abstract}
$\underline{\text { Abstract }}$
In this paper define the contractible edge eulerian graph that, let $\mu$ is a class of Eulerian graphs $G \in \mu$, the edge e in $G$ is called contractible edge eulerian graph if $G * e \in \mu$. The necessary conditions for Eulerian graphs to have contractible edge eulerian have been introduced, further, the even and odd contractible edge eulerian graph have been studied, we also define the contractible edge eulerian graph class, the edge e in $\mathrm{G}$ is satisfied property contraction is called contractible edge eulerian if $G * e \in \mu$. Tutte [7] proved every 3-connected graph non isomorphic to $k_{4}$ have 3-contractible and proved every 3connected graph on more than four vertices contains an edge whose contraction yield a new 3-connected graph [7]. We proved graph $\mathrm{G}$ is eulerian graph has contractible edge if non isomorphic to $k_{4}$. How over every 4-connected graph on at least seven vertices can be reduced to smaller 4-connected graph by contraction one or two edge subsequently [7]. Also we discussed the graph $G$ is eulerian on at least seven vertices can be contraction and saved the properties of eulerian graph. Let $G$ be a regular graph and eulerian graph, the edges e in $G$ is called contractible regular-eulerian graph if $G * e$ is regular-eulerian grah, We discussed relation contraction of eulerian-regular graph then $G$ has contractible if $d(v)=2$, if $d(v)>2$ then $G$ has not contractible regular-eulerian.
\end{abstract}




\section{University of Thi-Qar Journal Vol.11 No.4 DEC 2016}

Web Site: https://jutq.utq.edu.iq/index.php/main Email: journal@jutq.utq.edu.iq

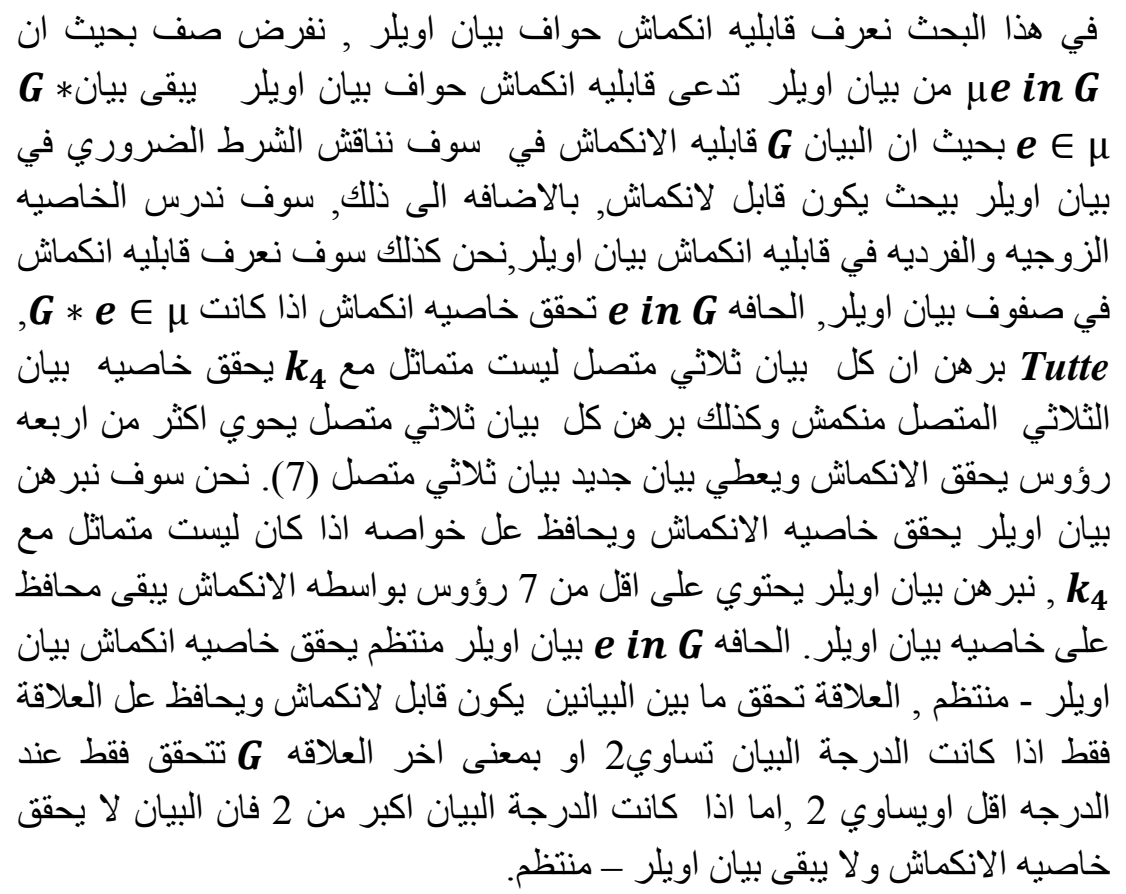

Key words: contractible edge eulerian graph, connected graph, eulerian graph. regular graph.

\section{Introduction}

All graphs considered here are simple and finite. For notation and terminology not given here. In this paper we shall study the contractible edge Eulerian in Eulerian graph. First, we give the definition of contractible edge Eulerian graph. Let $G$ be an Eulerian graph and e an edge of $G$. Consider the graph contractible $G * e$ obtained by contraction the edge e from $G$. If $G * e$ has vertices even degree and connected graph, we do the following contraction on $G * e$. For all vertices have even degree in $G * e$ and connected graph then completely eulerian graph. Second, we give definition of relation Eulerian graph with regular graph, let $G$ be an Eulerian graph then $G$ is not contractible regular-Eulerian graph. Thus e is contractible regular - Eulerian graph if $d(v)=2$, if $d(v)>2$ then $G$ has non contractible regular-Eulerian graph. 


\section{University of Thi-Qar Journal Vol.11 No.4 DEC 2016}

Web Site: https://jutq.utq.edu.iq/index.php/main Email: journal@jutq.utq.edu.iq

Let $\mathrm{e}=(\mathrm{u} ; \mathrm{v})$ be an edge of a graph $G=(V ; E)$. A contraction of the edge e consists of eliminating e and merging the vertex $u$ and vertex $\mathrm{v}$ into new vertex $\mathrm{w}$. The new graph $G^{\prime}=(\mathrm{V} \backslash\{\mathrm{u}$, $v\} \cup[w\}, E \backslash\{e\}) .[3]$

As following some definition, if for some positive integer $k$, $d(v)=k$ for every vertex $v$ of the graph $G$, then $G$ is called $k-$ regular graph. [1]

A vertex $u$ is said to be connected to a vertex $v$ in a graph $G$ if there is a path from $u$ to $v \cdot[1]$

A graph $G$ is called Eulerian or Euler if it has Euler tour.[1] Every eulerian graph is regular, but the converse is not true.

In this paper we define contractible edge eulerian graph as following.

Definition 1.1: Let $\mu$ a class of eulerian graphs, $G \in \mu$, the edge e in $\mathrm{G}$ is satisfied property contraction is called contractible edge eulerian if $G * e \in \mu$.

Definition 1.2: Let $G$ be a regular graph and eulerian graph, the edges e in $G$ is called contractible regular-eulerian graph if $G * e$ is regular- eulerian graph.

The following statements are equivalent for a connected graph $G$.

Theorem 1.3 [1]: A connected graph $G$ is euler if and only if the degree of every vertex is even. Tutte was the first who studied such method systematically for 2-connected graph and 3connected graph, with the following version of his celebrated Wheel Theorem [7].

Theorem 1.4 [7]: Every 3-connected simple graph non isomorphic to Wheel can be reduced to smaller 3-connected simple graph by either deleting or contraction an edge. Let us mention Negami's splitter theorem for 3-connected graph [5]. 


\section{University of Thi-Qar Journal Vol.11 No.4 DEC 2016}

Web Site: https://jutq.utq.edu.iq/index.php/main Email: journal@jutq.utq.edu.iq

Theorem 1.5 [5]: Let $\mathrm{H}$ be any 3-connected graph non isomorphic to wheel graph, then every 3-connected simple graph non isomorphic to $\mathrm{H}$ and contains a minor can be reduced to smaller 3-connected by deleting or contraction single edge.

Tutte proved every 3-connected graph non isomorphic to $k_{4}$ have 3-contractible and proved every 3-connected graph on more than four vertices contains an edge whose contraction yield a new 3-connected graph [7].

How over every 4-connected graph on at least seven vertices can be reduced to smaller 4-connected graph by contraction one or two edge subsequently [7].

Prove K. Ando and W. McCuiag [2, 6] as following.

Theorem 1.6 [2, 6]: Let $G$ be 3-connected graph with at least five vertices then $G$ has at least $|v(G)| \backslash 2$ contractible edge.

Also prove R, Halian of 3-connected graph.

Theorem 1.7 [4]: Let $G$ be minimally 3-connected graph and let $e=x y$ be an edge of $G$ if $\operatorname{deg}(x) \geq 4, \operatorname{deg}(y) \geq 4$, then e is contractible.

Prove Tutte of 2-connected has 2 contractible edges.[7]

Theorem 1.8 [7]: Every vertex 2-connected non isomorphic to $k_{3}$ is incident with 2 contractible edges.

Also prove Tutte of 3-connected graph.[7]

Theorem 1.9 [7]: Every 3-connected non isomorphic to $k_{4}$ can be reduced to smaller 3-connected graph by contraction.

2- Contractible edge in Eulerian graph. 


\section{University of Thi-Qar Journal Vol.11 No.4 DEC 2016}

Web Site: https://jutq.utq.edu.iq/index.php/main Email: journal@jutq.utq.edu.iq

In this section we study characterization of contractible eulerian graph. In the following result of eulerian graph, we proved that the eulerian graph has contractible.

Theorem 2.1: Let $G$ be an eulerian graph with $v(G) \geq 5$, then $G$ has at least $|v(G)| \backslash 2$ contractible edge eulerian.

Proof: Let $G$ be an eulerian graph with $v(G) \geq 5$, by theorem $1.3, G$ is a connected graph, every vertex has even degree. To prove $G$ contains contractible edge eulerian, we must prove:

1- $G$ contains edge such that $G * e$ is connected.

2-The degree of every vertex in $G * e$ has even degree.

As $G$ be connected graph with $\sum v(G) \geq 5$, if the number vertices equal to 5 , there exist

Path from $v_{1}$ to $v_{5}$.

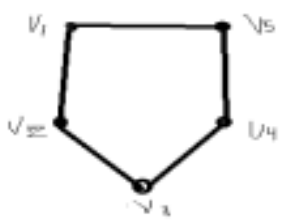

Graph 1

$$
p=v_{1} e_{1} v_{2} e_{2} v_{3} e_{3} v_{4} e_{4} v_{5} e_{5}
$$

Were contraction of second edge of path $p$, we get path connected. If $\sum v(G) \geq 5$, let the number vertices 10 , there exist path from $u$ to $u_{10}$. after contraction the number vertices is 9 vertices, then there exist path connected. We repeat method contraction of edges of each once to get at least $|v(G)| \backslash 2$. Then $G * e$ has at least $|v(G)| \backslash 2$ is connected graph and (1) hold. To establish (2), consider the edge e in $G, e=(u, v)$ in $G$, we get e contains of eliminating e and merging the vertex $u$ and vertex $v$ together into new vertex $w$, since $G$ is eulerian graph and with $v(G) \geq 5$ such that vertex $u$ and vertex $v$ has even degree. Thus 


\section{University of Thi-Qar Journal Vol.11 No.4 DEC 2016}

Web Site: https://jutq.utq.edu.iq/index.php/main Email: journal@jutq.utq.edu.iq

after contraction edge added degree $u$ with degree $v$ into vertex $w$,

$$
\begin{gathered}
d(w)=d(v)+d(u), \sum v(G) \geq 5, \\
|v(G)| \geq 5, d(w)=2 n, \\
|v(G * e)| \geq 4, \div 2 \\
\text { Then }|v(G * e)| \backslash 2 \geq 2
\end{gathered}
$$

Thus every vertex of $G * e$ has even degree, and (2) holds, and then $G$ has at least $|v(G)| \backslash 2$ contractible edge eulerian.

For each $n \geq 4$, the Wheel graph, with $\mathrm{n}$ vertices, is defined to be the the join $k_{1}+C_{n-1}$, of an isolated vertex with a cycle of length $n-1$. [1]

We defined isomorphism graph, An isomorphism from a simple graph $G$ to simple graph $\mathrm{H}$ is bijection $f: V(G) \rightarrow V(H)$ such that $u v \in E(G)$ if and only if $f(u) f(v) \in E(H)$.[1]

We introduce contraction of eulerian graph non isomorphic to wheel graph.

Theorem

2.2: Every eulerian simple graph non isomorphic to wheel graph $w_{5}$ then $G$ has smaller eulerian graph by contraction an edge.

Proof: Suppose that eulerian simple graph, as $G$ is eulerian graph. Then $G$ is connected and every vertex has even degree, by theorem 1.3 .

Since $G$ is connected simple graph and non- isomorphic to wheel graph $w_{5}$ then $\mathrm{G}$ has $(x, y)$ - path, we contraction of $(x, y)$-path of $\mathrm{G}$ is remain is connected graph, then $G * e$ is connected graph.

To prove $G * e$ has even degree of each vertex in. we must prove each vertex of $G * e$ after working contraction has even degree. Since $G$ non isomorphic to wheel graph, wheel graph is cycle of n-1 pulse complete graph $k_{1}$ and degree of each vertex of wheel has odd degree if we contraction edge of wheel, we get 


\section{University of Thi-Qar Journal Vol.11 No.4 DEC 2016}

Web Site: https://jutq.utq.edu.iq/index.php/main Email: journal@jutq.utq.edu.iq

graph has odd degree and graph none connected. Thus $G$ has even degree. consider the edge e in $G, e=(u, v)$ in $G$, we get e contains of eliminating e and merging the vertex $u$ and vertex $v$ together into new vertex $w$, since $G$ is eulerian graph such that vertex $u$ and vertex $v$ has even degree. Thus after contraction edge added degree $u$ with degree $v$ into vertex $w$ has even degree. Thus every vertex of $G * e$ has even degree, then $G * e$ is smaller eulerian graph.

Let $\mathrm{H}$ be a graph with a vertex set $\mathrm{V}(\mathrm{H})$ and an edge set $\mathrm{E}(\mathrm{H})$, then we say that $\mathrm{H}$ is subgraph of $G$ if $V(H) \subseteq V(G)$ and $E(H) \subseteq E(G)[1]$.

Theorem 2.3: Let $\mathrm{H}$ be an eulerian graph non isomorphic to wheel graph, then every eulerian simple graph non isomorphic to $\mathrm{H}$ can be reduced to smaller eulerian by contraction single edge.

Proof: Suppose that $G$ be eulerian simple graph, as $G$ is eulerian graph, then $G$ is connected and every vertex has even degree, by theorem (1.3). Since $G$ is connected simple graph. A graph $G$ non isomorphic to a subgraph $\mathrm{H}$ and a subgraph $\mathrm{H}$ non isomorphic to wheel graph, if $e=x y \in E(G)$ end points. We contraction of edge e so get smaller path of $\mathrm{G}$. Then $\mathrm{G} *$ e is connected graph. To prove $G * e$ has even degree of each vertex in $G * e$ by contraction single edge. Since $G$ non isomorphic to a subgraphs $\mathrm{H}$, thus $\mathrm{H}$ have vertices set is subset of vertices set of $G$ and edge set of $\mathrm{H}$ is subset of edge set of $G$, in other word $V(H) \subseteq$ $V(G)$ and $E(H) \subseteq E(G)$. Thus $G$ is non wheel and degree of each vertex of $G$ has even degree if we contraction single edge of $G$. Thus $G$ has even degree. consider the edge e in $G, e=$ $(a, b)$ in $G$, we get e contains of eliminating e and merging the vertex $a$ and vertex $b$ together into new vertex $c$, since $G$ is eulerian graph such that vertex $a$ and vertex $b$ has even degree. Thus after contraction edge added degree $a$ with degree $b$ into vertex $c$ has even degree. Thus every vertex of $G * e$ has even degree, then $G * e$ is smaller eulerian graph.

So introduce of the following result of eulerian graph. 


\section{University of Thi-Qar Journal Vol.11 No.4 DEC 2016}

Web Site: https://jutq.utq.edu.iq/index.php/main Email: journal@jutq.utq.edu.iq

Theorem 2.4: Every eulerian simple graph non- isomorphic to complete graph $k_{4}$ has contractible edge eulerian.

Proof: Suppose that $G$ be eulerian simple graph, we first prove $G * e$ is connected graph. Since $G$ is eulerian graph, by theorem (1.3), we must get $G$ is connected graph, Let e $\in E(G), e=u v$. There exist path from $\mathrm{x}$ to $\mathrm{y}$. by contraction an edge e remaining there exist path from $\mathrm{x}$ to $\mathrm{y}$. Then $G * e$ is connected graph. To prove $G * e$ each vertex has even degree, since $G$ is not isomorphic to $k_{4}$ has $n(n-1) \backslash 2$ edges, Such that every vertex of $k_{4}$ has 3 edge. Thus $k_{4}$ has odd degree of each vertex, the graph $G$ has even degree of every vertex because $G$ is eulerian graph. Since degree of the first vertex with degree of the second vertex applied of degree of new vertex by 2 . That is every vertex has 2 degree, thus $G * e$ is has contractible edge eulerian.

We prove the following strengthened of theorem ( 2.4 ) for eulerian graph.

Theorem 2.5: Every eulerian simple graph non isomorphic to $k_{2 n}$ has contractible edge eulerian.

Proof: Let $G$ be an eulerian simple graph, by using theorem 1.3, then $G$ is connected graph, we contract an edge of eulerian simple graph, let path of graph $G$ from u to $u_{n}$, such that $p=u e_{1} u_{1} e_{2} u_{2} e_{3} u_{3} \ldots u_{n}$. To contraction of $e_{2}$ in $G$, merging $u_{1}, u_{2}$ of new vertex $\mathrm{w}$ and deleting $e_{2}$ and repeat the method, we get path connected from $u$ to $u_{n}$.

Such that $p *=u e_{1} w e_{3} u_{3} \ldots . u_{n}$, in the following of graph 2

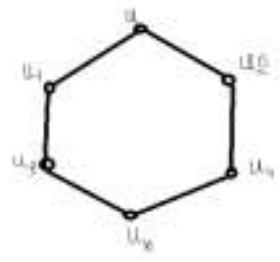




\section{University of Thi-Qar Journal Vol.11 No.4 DEC 2016}

Web Site: https://jutq.utq.edu.iq/index.php/main Email: journal@jutq.utq.edu.iq

\section{Graph 2}

There exists path after contraction an edge. Thus $G * e$ is connected graph. To prove $G * e$ has even degree of each vertex, since $G$ is not isomorphic to $k_{2 n}, k_{2 n}$ has $n(n-1) \backslash 2$ edges,

Such that every vertex of $k_{2 n}$ has 2 n- 1 edge. Thus $k_{2 n}$ has odd degree of each vertex. The graph $G$ has even degree of every vertex because $G$ is eulerian graph. To contract even complete graph introduced multiple edges. Thus contradiction with suppose that graph be simple is not multiple edge or loop graph. Since degree of the first vertex with degree of the second vertex applied of degree of new vertex is $2 \mathrm{n}$. That is every vertex has 2 n degree, thus $G * e$ is eulerian graph, and thus has contractible edge eulerian.

We prove every eulerian simple graph on seven vertices has contractible.

Theorem 2.6: Every eulerian simple graph has seve vertices non isomorphic to $k_{7}$ has contractible edge eulerian by 2 edges or single.

Proof: Let $\mathrm{G}$ bean eulerian simple graph has seven vertices non isomorphic to $k_{7}$, as $G$ is eulerian graph, by theorem 1.3 , we have $\mathrm{G}$ is connected graph and every vertex of $\mathrm{G}$ has even degree. Let vertices of graph $\mathrm{G}, v, u, x, y, z, w, t, \sum v(G)=7$, Since $G$ is connected graph, there exists path from $v$ to $t$. Thus $G * e$ has path connected from $v$ to $t$ after contraction a single edge, then $G * e$ is connected simple graph. To prove $G * e$ has even degree of each vertex, since $G$ is eulerian graph such that every vertex as even degree. Such that $\mathrm{G}$ non isomorphic to $k_{7}$, to working contraction of $k_{7}$ so get on multiple edge and we have a contradiction with suppose that $G$ is simple graph let $\sum v(G)=7$, we now introduce contraction of the first edge $e_{1}$. 


\section{University of Thi-Qar Journal Vol.11 No.4 DEC 2016}

Web Site: https://jutq.utq.edu.iq/index.php/main Email: journal@jutq.utq.edu.iq

$$
\left.G * e_{1}=(V \backslash(u, x)\} \cup\left\{w_{1}\right\}, E \backslash\left\{e_{1}\right\}\right) .
$$

such vertex $u$ has even degree and vertex $x$ has even degree after contraction merging $u$ and $x$ in new vertex $w_{1}$, thus vertex $w_{1}$ has even degree.

To contract of the second edge $e_{2} . \quad G * e_{2}=(V \backslash(y, z)\} \cup$ $\left.\left\{w_{2}\right\}, E \backslash\left\{e_{2}\right\}\right)$.

Such that vertex $y$ has even degree and vertex $z$ also has even degree after contraction merging $y$ and $z$ in new vertex $w_{2}$, thus vertex $w_{2}$ has even degree. Then every vertex of $G * e$ have even degree, thus $G * e$ is eulerian simple graph. Then $G$ has contractible edge eulerian by two edges.

Theorem 2.7: Let $G$ be eulerain simple graph non isomorphic to odd complete graph $k_{2 n+1}$ then $G$ have contractible edge eulerian.

Proof: Let $G$ be eulerain simple graph, since $G$ is eulerian graph, by theorem 1.3, $\mathrm{G}$ is connected graph and every vertex of $\mathrm{G}$ has even degree. As $G$ not isomorphic to $k_{2 n+1}$, in other word there exist not bijection from $f: G \rightarrow k_{2 n+1}$, such that, Let vertices $v_{1}, v_{2}, v_{3}, v_{4} v_{5}, v_{6}, v_{7} \ldots v_{n}$,

if $G$ connected non isomorphic to $k_{2 n+1}$, that is particular, since $G$ is connected simple graph, there exist path from $v_{1}$ to $v_{n}$, When applying contraction of edge $e_{1}=\left(v_{1}, v_{2}\right)$, such that a new graph remaining connected. There exist path connected. Then $G * e$ is connected simple graph. Since $G$ is not isomorphic to $k_{2 n+1}, k_{2 n+1}$ have $n(n-1) \backslash 2$ edges, such that every vertex of $k_{2 n+1}$ has even degree. We do not contraction of odd complete graph $k_{2 n+1}$ introduce multiple edge when working contraction. Since degree of the first vertex with degree of the second vertex applied of degree of new vertex is $2 \mathrm{n}$. That is every vertex has 2 n degree, thus $G * e$ has contractible edge eulerian graph. 


\section{University of Thi-Qar Journal Vol.11 No.4 DEC 2016}

Web Site: https://jutq.utq.edu.iq/index.php/main Email: journal@jutq.utq.edu.iq

Theorem 2.8: Let $G$ be an eulerian simple graph such that $\operatorname{deg}(v) \geq 2$ and $G$ contains 2 n- regular graph, the number vertices of $G$ is $V(G) \geq 4$, then $G$ has contractible edge eulerian.

Proof: Let $G$ be an eulerian simple graph such that $\operatorname{deg}(v) \geq 2$ and $G$ contains 2 n-regular

graph the number vertices of $G$ is $V(G) \geq 4$, since $G$ is eulerian graph, by theorem

$1.3, G$ is connected graph and every vertex have even degree.

When working contraction of graph containing 2n-regular graph such that the first probability

if $\operatorname{deg}(v)=2$ and $v(G)=4$, suppose that $u_{1}, u_{2}, u_{3}, u_{4}$, degree of each vertex is 2 after working contraction we get the number vertices is 3 and degree remaining is 2 ,

There exist path from $u_{1}$ to $u_{3}$. Thus the graph $G * e$ is connected. That is each vertex has even degree. Then $G * e$ is eulerian graph.

The second probability if $\operatorname{deg}(v)>2$ and $\mathrm{v}(\mathrm{G})>4$, let the number vertices is 5 , suppose that $u_{1}, u_{2}, u_{3}, u_{4}, u_{5}$, degree of each vertex is 4 because the graph contains $2 n$-regular taking the integer even after working contraction so get the number vertices is 4 and degree remaining is 4 , There exist path from $u_{1}$ to $u_{4}$. Thus the graph $G * e$ is connected. Such that each vertex has even vertex. Then $G * e$ is eulerian graph.

The third probability if $\operatorname{deg}(v)=2$ and $\mathrm{v}(\mathrm{G})>4$, let the number vertices is 5 or greater than 5 , taking $v(G)=5$, suppose that $u_{1}, u_{2}, u_{3}, u_{4}, u_{5}$, degree of each vertex is 2 because degree equal to 2 taking the integer even after working contraction we get the number vertices is 4 and degree remaining is 2 , There exist path from $u_{1}$ to $u_{4}$. Thus the graph $G * e$ is connected. 


\section{University of Thi-Qar Journal Vol.11 No.4 DEC 2016}

Web Site: https://jutq.utq.edu.iq/index.php/main Email: journal@jutq.utq.edu.iq

Such that each vertex has even vertex. Then $G * e$ is eulerian graph.

The fourth probability if $\operatorname{deg}(v)>2$ and $\mathrm{v}(\mathrm{G})=4$, let the number vertices is 4 , suppose that $u_{1}, u_{2}, u_{3}, u_{4}$, degree of each vertex is 4 because the graph contains 2 n-regular taking the integer even after working contraction so get the number vertices is 3 and degree remaining is 4 , There exist path from $u_{1}$ to $u_{3}$. Thus the graph $G * e$ is connected. Such that each vertex has even vertex. Then $G * e$ is eulerian graph. Thus $\mathrm{G}$ has contractible edge eulerian.

We prove relation of contraction of eulerian graph with regular graph.

Theorem 2.9: Let $G$ be an eulerian graph-regular and nonisomorphic to complete graph $k_{3}$, and let $d(v)=2$, then $G$ has contractible regular -eulerian graph.

Proof: Suppose that $G$ be eulerian graph , by theorem 1.3, thus $\mathrm{G}$ is connected graph and each vertex has even degree, in other word $d(v)=2 n, \mathrm{n}=1,2,3,4, \ldots \ldots$, of each vertex. From theorem 2.7 then $G$ has contractible edge eulerian graph. to prove $G * e$ is regular-eulerian graph, as $\mathrm{G}$ non isomorphic to complete graph $k_{3}$, From Definition 1.2, she must show that $G * e$ have degree equal of each vertex in graph G. since $\mathrm{G}$ has $d(v)=2 n$ of each vertex, from let $\mathrm{d}(\mathrm{v})=2$. Let $n=1$, then $d(v)=2$, thus we contraction of one edge that pass of vertex once such that each vertex $G * e$ is 2 - regular graph. then $G * e$ is regular-eulerian then $G$ has contractible regular-eulerian gr $\square$ h.

Hint: if applying theorem 2.9 and non- isomorphic to complete graph $k_{4}$, If $\mathrm{n}=2$ then $\mathrm{d}(\mathrm{v})=4$, we contraction of edge in $G$ thus the new graph if multiple edge we contravention simple graph and non- isomorphic to complete graph $k_{4}$, we cannot contraction of one edge is not get regular graph and eulerian graph. We is not relation of contraction of regular graph with 


\section{University of Thi-Qar Journal Vol.11 No.4 DEC 2016}

Web Site: https://jutq.utq.edu.iq/index.php/main Email: journal@jutq.utq.edu.iq

eulerian graph wither more cases is not relation of contraction of regular graph with eulerian graph.

We discuss is not relation contraction of regular graph and eulerian graph if $\mathrm{d}(\mathrm{v})>2$.

Theorem 2.10: Let $G$ be an eulerian graph and $(v)>2, \forall v$, then $G$ has no contractible regular-eulerian graph if contraction of one edge.

Proof: Let $G$ be an eulerian graph and $d(v)>2, \forall v$, if $d(v)=3$, we contraction of one edge of graph then new graph is not regular and is not eulerian graph then $G$ has not contractible edge, if we contraction of edges from all vertex of graph then graph is not simple such that degree is even and equal of each vertex. Since we cannot call contractible because suppose that the graph is simple.

let $d(v)=4$, since $G$ is eulerin graph, each vertex has even degree, to contraction of once edges so get vertex has $d(v)<4$, but edges anther have degree 4 . That other vertex have degree different, thus $\mathrm{d}(\mathrm{v})$ is not equal 4 Thus $\mathrm{G}$ is not regular and not eulerian graph. Then $G$ has no contractible edge. If we contraction of each edges of graph such that $d(v)=4$, then new graph $G * e$ is regular but degree of graph is odd such that the new graph is not eulerian graph. also degree greater 4 , we cannot relation of regular - eulerian graph.

\section{Conclusions}

1-Let $G$ be eulerian graph with $v(G) \geq 5$, then $G$ has at least $|v(G)| \backslash 2$ contractible edge eulerian.

2 - Every eulerian simple graph non isomorphic to wheel graph $w_{5}$ then $G$ has smaller eulerian graph by contraction an edge.

3-Every eulerian simple graph non isomorphic to $k_{2 n}$ then $G$ has contractible edge eulerian. 
University of Thi-Qar Journal Vol.11 No.4 DEC 2016

Web Site: https://jutq.utq.edu.iq/index.php/main Email: journal@jutq.utq.edu.iq

4-Let $G$ be eulerain simple graph non isomorphic to odd complete graph $k_{2 n+1}$ then $G$ have contractible edge eulerian.

5-Let $G$ be an eulerian graph and $(v)>2, \forall v$, then $G$ has no contractible regular-eulerian graph if contraction of one edge.

6- Let $G$ be an eulerian graph-regular and non- isomorphic to complete graph $k_{3}$, let $d(v)=2$, then $G$ has contractible regular -eulerian graph.

7- Let $G$ be an eulerian simple graph such that $\operatorname{deg}(v) \geq 2$ and $G$ contains 2 n-regular graph, the number vertices of $G$ is $V(G) \geq 4$, then $G$ has contractible edge eulerian.

8- Let $\mathrm{H}$ be any eulerian graph non isomorphic to wheel graph, then every eulerian simple graph non isomorphic to $\mathrm{H}$ can be reduced to smaller eulerian by contraction single edge. 
University of Thi-Qar Journal Vol.11 No.4 DEC 2016

Web Site: https://jutq.utq.edu.iq/index.php/main Email: journal@jutq.utq.edu.iq

References

(1)- Clark. J. and Holton D. A., A First look at Graph Theory, Department of mathematics and statics of Otage New Zealand, (1961).

(2)- K. Ando, H. Enomoto \& A. Saito, Contractible Edge in 3-connected Graphs, J. combin. Theory ser. B42 (1982).

(3)- Paul Van Dooren, Graph Theory and Applications , Université catholique de Louvain, Louvain-la-Neuve, Belgium Dublin, August 2009.

(4)- R,Halin, Zur Theoriedern n-fach Zusammenhangenden Graplen, Abh, Math.Sem. Uinv. Hamburg 33 (1969), 133164.

(5)- Seiya Negami. A characterization of 3-connected Graph contianing given Graph. J. Combin. Theory. Ser. B, 3260(2): 69.74, 1982.

(6)-W.McCuiag, Edge Contraction in 3-connected Graphs, Ars Combin. 29 (1990), 299-308.

(7)- W.T.Tutte, ATheory of 3-connected Graphs. Naderl. A kad.weten sch pro.ser. A 64 - indag. Math., 23:441- 455, 1961. 